OPEN ACCESS

Edited by:

Cathy Craig,

Queen's University Belfast, UK

Reviewed by:

Graziella Madeo,

University of Rome Tor Vergata, Italy

Maria Stamelou,

University of Athens, Greece

*Correspondence:

Joseph Jankovic

josephj@bcm.edu

Specialty section:

This article was submitted to Movement Disorders, a section of the

journal Frontiers in Neurology

Received: 17 September 2015

Accepted: 22 October 2015

Published: 11 November 2015

Citation:

Ashoori A, Eagleman DM and Jankovic J (2015) Effects of Auditory

Rhythm and Music on Gait Disturbances in Parkinson's Disease.

Front. Neurol. 6:234.

doi: 10.3389/fneur.2015.00234

\section{Effects of Auditory Rhythm and Music on Gait Disturbances in Parkinson's Disease}

\author{
Aidin Ashoori' , David M. Eagleman ${ }^{2}$ and Joseph Jankovic ${ }^{3 *}$ \\ ${ }^{1}$ Columbia University College of Physicians \& Surgeons, New York, NY, USA, ${ }^{2}$ Department of Neuroscience, Baylor College \\ of Medicine, Houston, TX, USA, ${ }^{3}$ Department of Neurology, Parkinson's Disease Center and Movement Disorders Clinic, \\ Baylor College of Medicine, Houston, TX, USA
}

Gait abnormalities, such as shuffling steps, start hesitation, and freezing, are common and often incapacitating symptoms of Parkinson's disease (PD) and other parkinsonian disorders. Pharmacological and surgical approaches have only limited efficacy in treating these gait disorders. Rhythmic auditory stimulation (RAS), such as playing marching music and dance therapy, has been shown to be a safe, inexpensive, and an effective method in improving gait in PD patients. However, RAS that adapts to patients' movements may be more effective than rigid, fixed-tempo RAS used in most studies. In addition to auditory cueing, immersive virtual reality technologies that utilize interactive computer-generated systems through wearable devices are increasingly used for improving brain-body interaction and sensory-motor integration. Using multisensory cues, these therapies may be particularly suitable for the treatment of parkinsonian freezing and other gait disorders. In this review, we examine the affected neurological circuits underlying gait and temporal processing in PD patients and summarize the current studies demonstrating the effects of RAS on improving these gait deficits.

Keywords: Parkinson's disease, gait, freezing, music, rhythm

\section{INTRODUCTION}

Gait disorders, particularly freezing of gait (FOG), are among the most disabling features of Parkinson's disease (PD) (1). Rhythmic auditory stimulation (RAS), such as listening to marching music, has been used to ameliorate this motor abnormality (2). The observation that sensory input, such as RAS, can help overcome freezing suggests that the motor program for gait is relatively intact in patients with PD but cannot be appropriately accessed without the sensory input (3). In this review, we will examine the role of auditory rhythm and music on parkinsonian gait.

...there's something about the temporal structure of the music, the emotional content of the music, that arouses areas of the brain that are still functioning and allows a lost ability to become present as they participate in the music. - Dr. Concetta Tomaino, Executive Director/Co-Founder, Institute for Music and Neurologic Function (4) 
A recent success story for music therapy from a neurological perspective is the speech recovery of Congresswoman Gabrielle Giffords after suffering a gunshot wound to the head in 2011. Giffords was unable to speak due to severe damage in the left hemisphere of her brain. However, remarkably, Giffords was able to sing parts of songs. After working for several years with music therapy, she slowly regained the natural rhythm of speech through vocalizing musical phrases (5). In Giffords's words, "music therapy was so important in the early stages of my recovery because it can help retrain different parts of your brain to form language centers in areas where they weren't before you were injured" (6). Through singing, Giffords's undamaged brain regions were able to rewire themselves to recover her ability to speak. Indeed, research has shown that music not only helps patients recover from stroke but may improve gait in patients with $\mathrm{PD}$, and learning to play a musical instrument may induce neuroplastic changes that may translate into improved motor and cognitive function (7). This was emphasized by the late Oliver Sacks in his book Musicophilia, entirely devoted to this topic (8). Rarely, however, playing a musical instrument may uncover underlying motor abnormality which becomes manifested as task-specific dystonia $(9,10)$.

Rhythmic stimulation through music and sound has been shown to improve motor deficits in a variety of movement disorders. Rhythm is defined as the time-based pattern of music or sound that consists of perceptible groupings of notes, beats, accents, and phrases (11). Beat is the unit of rhythmic pulse (11).

Tasks requiring melody perception and production recruit both the auditory and the motor areas of the brain (12-17). Passively listening to rhythmic stimuli, even in the absence of motor actions or intent, recruits the auditory systems as well as the mid-premotor cortex (PMC) and the supplementary motor area (SMA) (18). Through a process called rhythmic entrainment (19), humans naturally move in synchrony to external rhythmic cues. The evidence of rhythmic entrainment can be observed when humans spontaneously move or dance to the beats of music, even without being consciously aware of their action. However, rhythmic entrainment is not limited to auditory cues. As humans walk side by side, they naturally synchronize their footsteps without instruction or conscious intent (20-22). This bipedal locomotion relies on our innate internal timing, which may control our conscious and subconscious abilities to extract rhythm from the external world (23).

The strong connections between gait, innate internal timing, and rhythmic perception are demonstrated by humans' rhythmic preference in music. Although humans' perceptible temporal range is $40-300 \mathrm{bpm}(24-27)$, the preferred musical tempo is at 120-130 bpm (28). This preferred tempo is at the middle of the range of the average gait cadence of males (103-150 strides per minute) and females (100-149 strides per minute) across different age groups (29). Accordingly, humans' natural musical rhythmic preferences may have been influenced by their natural spontaneous gait rhythm. This powerful connection between rhythm and locomotion has led rhythmic entrainment to be clinically employed for gait rehabilitation in patients with neurological disorders including stroke, traumatic brain injury, cerebral palsy, and PD $(7,19)$. Rhythmic entrainment through music tempo has also been used to improve running cadence (30), which may be beneficial in preventing injuries in runners with PD or in athletes with "runner's dystonia" (31).

\section{GAIT IMPAIRMENTS IN PARKINSON'S AND CURRENT THERAPIES}

Parkinson's disease, the second most common neurodegenerative disorder (32), is a complex neurological disorder that negatively impacts both motor and non-motor functions (33). The disease is caused by the degeneration of dopaminergic (DA) neurons in the substantia nigra associated with neuronal inclusions called Lewy bodies, leading to DA deficiency in the basal ganglia (BG) (34). This deficiency results in four cardinal symptoms of $\mathrm{PD}$ that can be remembered by the tremor at rest, rigidity, akinesia (or bradykinesia), and postural instability (33-35). These symptoms are often accompanied by gait impairments (36) that are particularly prominent in the postural instability gait difficulty (PIGD), in contrast to the tremor dominant, subtype of PD (37). Gait abnormalities become also more severe in the late-stage PD (38).

Gait disorders in PD are characterized by stooped posture, shuffling steps, flexed knees, narrow base, reduced arm-swing, turning en bloc, and FOG, which is one of the most debilitating features of PD $(1,39)$. While walking, patients suddenly lose the ability to lift their feet and become stuck in place for several seconds or even minutes despite their efforts to initiate forward movement (40). FOG can be provoked by perceived obstructive environmental cues, such as attempting to walk through narrow doorways. Compared to healthy adults, PD patients have a shorter stride length, slower velocity, and more unpredictable fluctuations between consecutive strides (1, 38, 41-46). Indeed, FOG has been shown to be associated with marked disruption to internal rhythmic timing (47). Table 1 lists and summarizes the basic parameters used to measure the quality of gait.

Emergence of gait abnormalities often indicates a poor prognosis for PD patients as they correlate with bradykinesia, rigidity, and cognitive impairment associated with cortical Lewy bodies $(36,48)$ and leads to more frequent falling, a major cause of death among patients with PD (1). Several studies have shown that FOG in patients with $\mathrm{PD}$ correlates with poor quality of life, disease severity, apathy, and exposure to anticholinergic drugs; it may, but not always, improve with DA therapy (49).

TABLE 1 | Basic parameters of gait and their definitions and units of measurement.

\begin{tabular}{|c|c|}
\hline Gait parameter & Definition \\
\hline Walking speed (m/s) & Distance walked per unit of time \\
\hline Cadence (steps/min) & Number of steps per unit of time \\
\hline Stride time (s) & $\begin{array}{l}\text { Time between two successive ground contacts } \\
\text { of the same foot }\end{array}$ \\
\hline Stride length (m) & $\begin{array}{l}\text { Distance covered between two successive ground } \\
\text { contacts of the same foot }\end{array}$ \\
\hline Step time (s) & $\begin{array}{l}\text { Time between two successive ground contacts } \\
\text { of the opposite feet }\end{array}$ \\
\hline Step length (m) & $\begin{array}{l}\text { Distance covered between two successive ground } \\
\text { contacts of the opposite feet }\end{array}$ \\
\hline
\end{tabular}


The mechanisms of PD-related gait disorders, and FOG in particular, are not well understood. Impaired functional connectivity between the BG and the dorsolateral prefrontal cortex and the posterior parietal cortex has been suggested by recent connectivity studies $(50,51)$. Although DA deficits clearly play an important role in gait disturbances associated with PD, FOG often does not respond well to DA therapy, suggesting extranigral pathology in this particular gait disorder. In a cross-sectional study involving $143 \mathrm{PD}$ patients using positron emission tomography imaging, patients with FOG had lower DA striatal activity, decreased neocortical cholinergic innervation, and greater neocortical deposition of $\beta$-amyloid compared to non-freezers (52).

Conventional therapeutic interventions for PD, such as pharmacotherapy and deep brain stimulation (DBS), can be effective for treating the cardinal motor symptoms but have shown limited efficacy in gait abnormalities (53). Levodopa, a DA precursor and one of the main pharmacotherapies of PD, has limited therapeutic effects on balance and gait disturbances (40). Furthermore, anti-PD medications may produce side effects including lightheadedness, drowsiness, and dyskinesias which can exacerbate gait abnormalities (1). Although DBS typically improves tremor, rigidity, bradykinesia, and levodopa-related motor complications (54), this therapeutic modality results in only minimal benefits in patients whose primary symptoms are $\operatorname{PIGD}(1,55,56)$.

\section{NEURAL MECHANISMS OF CUED GAIT TRAINING}

In recent years, there have been numerous studies demonstrating the therapeutic efficacy of RAS in gait abnormalities associated with PD. An increasing body of research suggests that PD involves a deficit in temporal processing (57) and that internal rhythmic timing is more disrupted among PD with gait deficits than among patients without gait deficits (47). It has been proposed that internal timing is dependent on striatal DA levels (58), and that timing problems may be a potential marker for frontal and striatal dysfunctions in PD (59). Accordingly, we hypothesize that the temporal deficits in PD are a major contributor to gait impairments. This is supported by the finding that DA replacement therapy reduces the timing deficits in PD (60), and that timing deficits are induced by changes in the expression levels of striatal D2 receptors (61). Furthermore, timing deficits are also found in other DA-related disorders including schizophrenia $(58,62,63)$.

To understand temporal dysfunction, one must consider the two fundamental modes of timing: explicit and implicit timings. Explicit timing is required to make deliberate estimates of duration and relies on internal sense of time (64). Implicit timing utilizes external cues and relies less on conscious time-based judgments, engaging automatic timing systems. An example of an implicit timing task is the serial prediction task, which requires the subject to use a regularly timed stimulus to make temporal predictions about future stimuli $(64,65)$. Patients with PD have greater difficulty with explicit timing than with implicit timing. More specifically, PD patients have problems with explicit temporal discrimination tasks involving tactile, visual, and auditory stimuli, and explicit timing performance decreases as disease severity increases (66-69). The underlying neural networks of implicit and explicit timing are distinct. While implicit timing mainly recruits the cerebellum and is less dependent on the BG and the SMA (70-72), explicit timing recruits the BG, the SMA, the PMC, and the cerebellum (73).

The BG-SMA-PMC network is directly involved in rhythm perception in the presence or absence of motor actions $(18,74$, $75)$. In this network, the dorsal striatum (caudate and putamen) of the BG serves the most crucial role since it generates the internal pacing required for time estimation $(73,76)$. Thus, the $\mathrm{BG}$ is directly involved in perceptual and motor timing (77-79). The D2 receptors in the striatum mediate the DA signaling that controls the speed of this internal pacing (80-85). The lack of DA innervation to the BG in PD causes slower internal pacing (76), which leads to impairments in motor and perceptual timing abilities $(17,69,72,86,87)$. In further support of the BG's crucial role in timing, non-PD patients with focal lesions in the BG have similar difficulty with motor rhythmic synchronizations and have difficulty adapting to tempo changes (88).

Given that gait and other motor deficits in PD are strongly associated with timing impairments, RAS is a promising strategy for gait rehabilitation. Although PD patients have impairments with external timing due to internal pacing dysfunction, patients still have the ability to make temporal predictions through implicit timing. In other words, PD patients can still use external rhythmic cues to inform temporal-based decisions, such as when the next footstep should occur. Since implicit timing is still mostly intact in PD patients, they compensate for the disruption in the BG-SMA-PMC (explicit timing) by recruiting the cerebellum (89) (essential for implicit timing).

Although internal pacing is disrupted in PD patients, this timing alteration can be corrected and recalibrated through motor-sensory interaction with the world $(3,90)$. Cued gait training utilizes the implicit timing abilities still present in PD patients to recalibrate the internal clock. In RAS, PD patients are instructed to walk while synchronizing their footsteps to the salient beats of the music or metronome. RAS can be combined with visual cues such as patterned tiles or stripes placed along the walkway for multisensory cueing.

The schema in Figure 1 summarizes the basic neural pathways involved in gait training. In the absence of external cueing, internal cueing signals generated by the BG-SMA-PMC circuit feed into the motor programs, which are carried out in the medial motor areas comprised of the SMA and the cingulate motor area (91). During locomotion, the spinocerebellar, the spinothalamic, the spinoreticular, and the spinohypothalamic tracts carry somatosensory information, such as proprioception back to the brain $(3,92)$. The information carried by the somatosensory feedback modulates the internal clock of explicit timing (62) in the BG-SMA-PMC circuit and helps plan and predict future cued motor tasks.

The motor programs of gait appear to be relatively intact in $\mathrm{PD}$ patients, but due to impaired internal timing, the programs cannot be easily accessed without external cues (1, 3, 33). External rhythmic cues include visual and auditory sensory stimuli and can serve as surrogate cues for the impaired internal timing (93, 94). Accordingly, auditory and visual stimuli can bypass the 


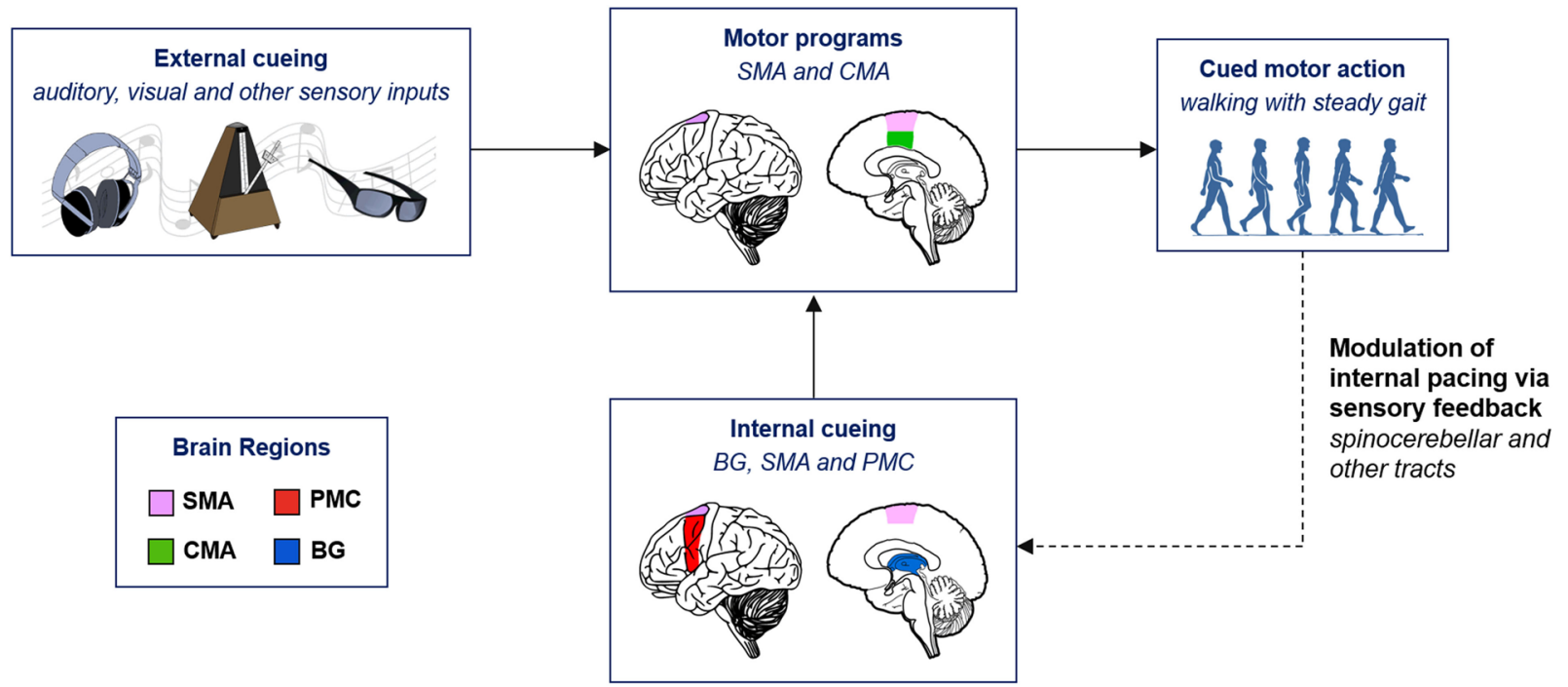

FIGURE 1 | Neurological schema of cued gait training. BG, basal ganglia; CMA, cingulate motor area; PMC, premotor cortex; SMA, supplementary motor area.

damaged BG and help the patients improve their gait by inducing motor-sensory feedback signals that recalibrate internal pacing. After the correct temporal scheme is re-established with RAS and potentiated through the BG-SMA-PMC circuit, patients can sustain improved locomotion for a period of time in the absence of external cueing. Gait rehabilitation through RAS has been recognized to benefit PD patients for almost two decades. In 1996, Thaut et al., using renaissance style instrumental music as the rhythmic cues, 3-week gait training with RAS significantly improved gait velocity, cadence, and stride length in PD patients (44). One year later, a similar study showed that RAS with cues that were $10 \%$ faster in tempo than the patients' baseline cadence had even a greater improvement on gait deficits (95). Since then there have been numerous reports on the effect of music- or metronome-based gait training in PD patients. Below, we will discuss some of the recent key studies on cued gait training to better understand the challenges of gait therapy and to formulate a future direction for RAS in PD.

\section{Optimal Auditory Cues for Gait Training}

Gait-training studies in PD patients have used either music or simple isochronous sounds, such as a metronome, as cues for RAS. Cue type can affect gait parameters differently depending on factors, such as the participants' health and age. Although there has not yet been a published direct comparison between music and metronome in gait rehabilitation in PD patients, several studies have done this with healthy participants. One study reports that healthy young adults walked faster with music than with metronome cues (96). Another similar study in healthy older adults (age $>65$ ) demonstrated that both music and metronome cues significantly increased cadence, but that only music significantly increased stride length and gait velocity (97). Contrary to these results in healthy participants, Huntington's disease patients walked faster when cued by the metronome rather than with music (98).
While the studies in healthy subjects suggest that cues with music are more effective than with a metronome at increasing gait velocities, a study by Leow et al. (99) reports that cues with a metronome rather than with music elicit better gait synchronization in healthy young adults. The same study further compares the effects of two types of music on gait: high-groove music (high beat salience) and low-groove music (low beat salience). Between these two types of musical cues, high-groove music elicited better gait synchronization and faster gait velocity. Low-groove music was not as effective, and even had a detrimental effect on gait in weak beat-perceivers (99). Music familiarity is also an important factor in RAS. RAS with familiar songs results in faster gait velocity and less stride variability than with unfamiliar songs. This is likely due to the fact that synchronizing footsteps to a familiar beat structure require less cognitive demand. Enjoyment of familiar music may also have had a role in eliciting a faster gait (100).

A variety of devices have been developed to provide customized fixed-temp RAS. Recently, a research group in Madrid, Spain (Brainmee ${ }^{\mathrm{TM}}$ ) developed Listenmee ${ }^{\circledR}$, an intelligent glasses' system, that employs RAS to improve gait (101). The glasses are portable and contain built-in headphones that allow the user to listen to isochronous (metronome-like) auditory cues while walking. The sounds are customizable to various styles, such as ambient, percussive, electronic, and vocal. The user controls the device via Bluetooth with the Listenmee ${ }^{\circledR}$ smartphone application. The research groups plan to turn the device into an auditory feedback system by integrating feedback to spatial movements. The device will include a built-in video camera and a laser emitter to assess motion in the visual field and provide responsive visual cueing. The group has yet to publish the results of the efficacy of this integrated visual and auditory feedback system.

An experiment showing the efficacy of the non-feedback device involved 10 PD patients between the ages of 45 and 65 years (101). Inclusion factors consisted of a history of frequent FOG and falling as well as failure to respond to medication and 
physical therapy. Five of the patients received DBS with minimal gait improvement prior to the study. In this study, patients were instructed to walk while off DA therapy. Cadence, stride length, and walking speed were measured with and without RAS. Patients showed significant improvement for all three gait parameters while listening to auditory cues.

\section{Musically Cued Gait Training: Sustained Benefits Beyond Gait Rehabilitation}

A recent study by Benoit et al. (102) shows that musically cued gait training significantly improves multiple deficits of PD, including in gait, motor timing, and perceptual timing. The study consisted of 15 non-demented patients with idiopathic PD (Hoehn and Yahr stage 2). The patients had no prior musical training and maintained their DA therapy regimen during the trials. There were three 30-min training sessions per week for 1 month. During each session, the participants walked to the salient beats of German folk music without explicit instructions to synchronize their footsteps to the beat. Compared to pretraining gait performance, the PD patients showed significant improvement in gait velocity and stride length during the training sessions. The gait improvement was sustained for 1 month post-training, indicating a lasting therapeutic effect for uncued gait.

This RAS training also significantly improved motor and perceptual timing. Pretraining, immediately post-training, and 1 month post-training, patients participated in a battery of motor and perceptual timing tests of duration discrimination, beat alignment, paced tapping, and adaptive tapping. Prior to training, $73 \%$ of the patients displayed timing deficit that decreased to $67 \%$ immediately post-training and only $40 \%$ 1 month post-training. Thus, in addition to gait, RAS improves perceptual timing with continued therapeutic effect even in the absence of auditory cueing. This study in the context of the previously mentioned study by Leow et al. suggests a circular relationship between rhythm perception and gait performance: improved beat perception increases the efficacy of gait training (99) and improved gait training increases beat perception ability (102) (Figure 2).

\section{Interactive Cueing Systems}

Although the efficacy of gait training with RAS has been proven, the rigid, fixed-tempo of the cues implemented by most studies has limited applications to PD patients. Fixed-tempo RAS requires increased demand for attention to synchronize footsteps with auditory cues, thus invoking higher-level cognitive processes (103). This can be problematic for PD patients, in whom multitasking while walking can trigger or exacerbate their gait difficulties (104-106). Even in healthy participants, fixed-tempo RAS can result in random and unpredictable stride intervals (107). Therefore, attempts have been made to improve RAS by integrating an adaptive system that provides feedback from human rhythm to determine cueing rhythm. A cueing system that aligns to the patients' movements would demand less attention, which may lead to greater gait improvements than with fixed, non-adaptive cueing (108).

WalkMate, an interactive RAS device developed by Yoshihiro Miyake and colleagues, was tested on 20 PD patients undergoing

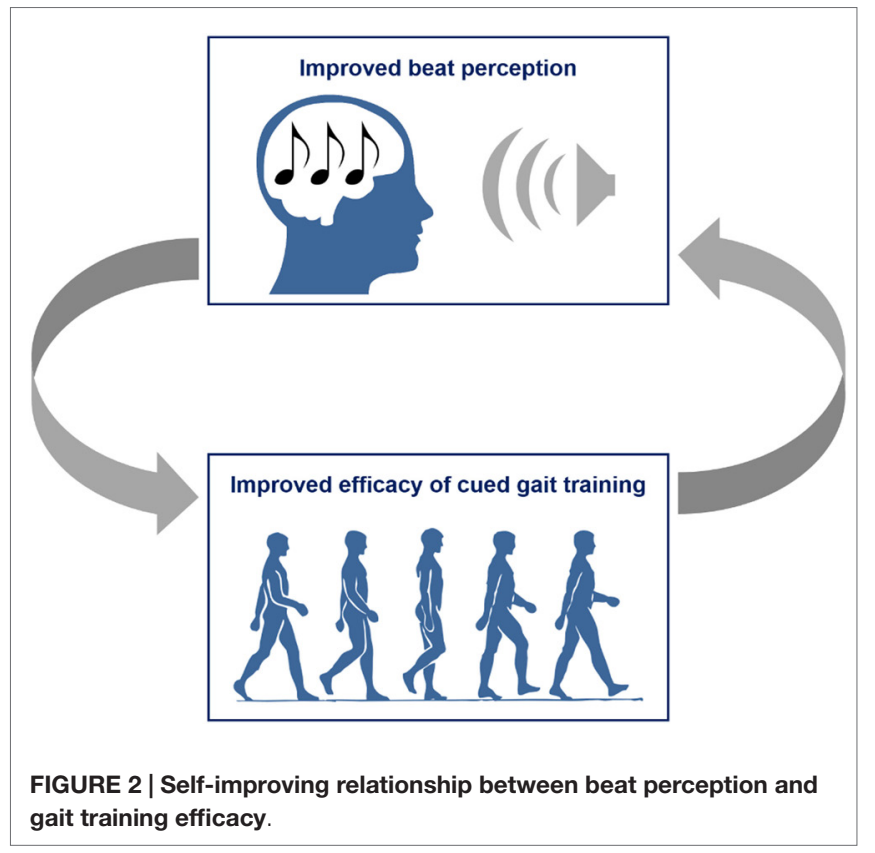

DA therapy and on 16 healthy controls (109). The device utilizes pressure sensors in the shoes that feed gait timing data into a computer system, and adjust the metronome cueing tempo in real-time. The efficacy of WalkMate on gait was compared with fixed-tempo RAS and a silence-control condition. Gait dynamics were analyzed using the detrended fluctuation analysis (DFA) fractal-scaling exponent, which is associated with gait adaptability and one of the best measures of predicting falling $(46,109,110)$. In a silent-control condition the PD patients had significantly lower fractal scaling (higher variability) in stride than the healthy subjects. During fixed-tempo RAS, PD patients' stride had even lower fractal scaling than during the silent-control condition, consistent with past findings (107). With WalkMate, PD patients' fractal scaling became significantly better than the silent-control condition and reached the DFA baseline of healthy subjects in the silent-control condition. Furthermore, gait improvement persisted in the absence of the adaptive WalkMate cues 5 min after the training sessions (Figure 3) (109).

More recently, a similar device named D-Jogger was tested on healthy subjects to study the synchronization of gait to adaptive rhythmic cues (111). D-Jogger is a music player that adjusts the musical tempo to the listeners' gait rhythm (Figure 4) (112). In the most effective adaptive strategy (out of the four adaptive strategies tested), the participant initially begins walking in the absence of music. The music then begins by the first beat matching the footfall and continues with a tempo equal to the average gait tempo sampled from the previous $5 \mathrm{~s}$. The results from healthy participants motivate further testing of D-Jogger on patients with $\mathrm{PD}$ or other movement disorders.

\section{Virtual Reality: A Potential for Combined Visual and Auditory Cueing}

In PD patients, locomotion and postural control have an increased dependence on perceptual vision $(113,114)$ that can 


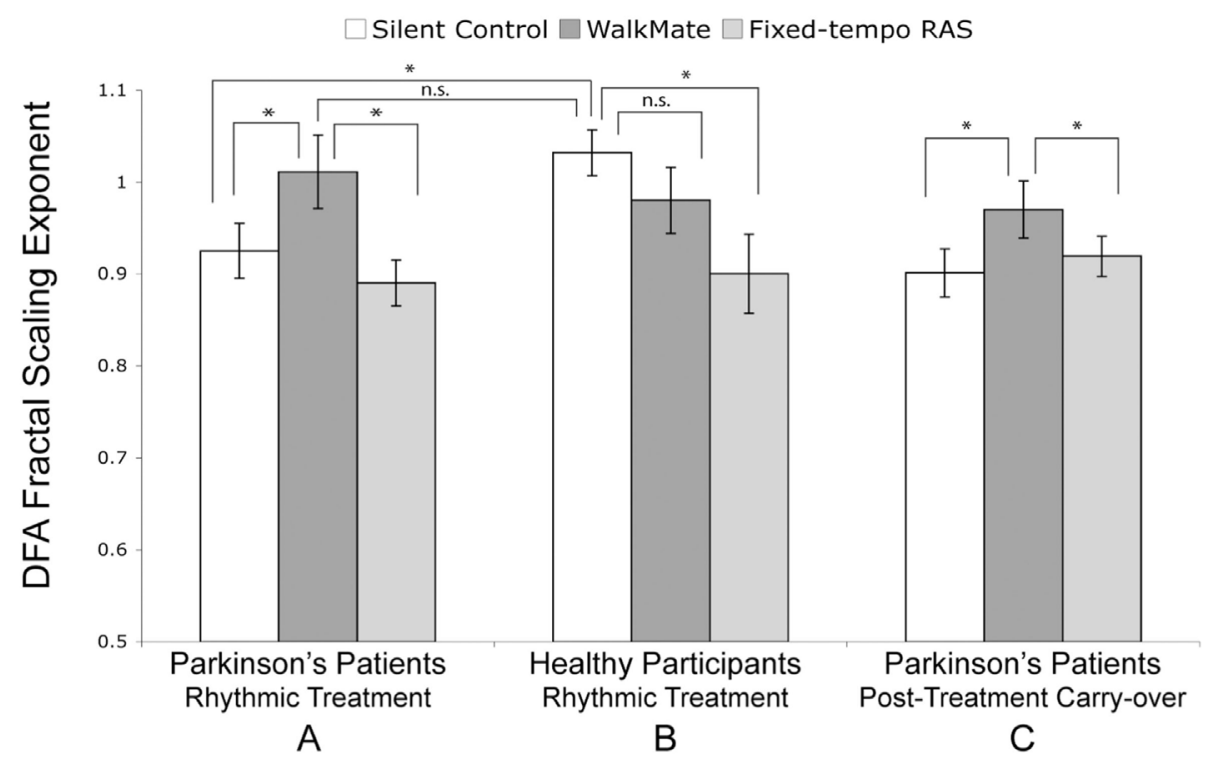

FIGURE 3 | Interactive rhythmic auditory stimulation using WalkMate. (A) Parkinson's patients during rhythmic treatment, (B) Healthy participants during rhythmic treatment, and (C) Parkinson's patients' carry-over effect during a silent trial 5 min after the rhythmic treatment. The cueing conditions are unassisted silent control, interactive WalkMate rhythmic auditory stimulation (RAS), and fixed-tempo RAS. Error bars represent six SEM. ${ }^{*} P<0.05$; n.s., non-significant. Reproduced from Hove et al. (109).

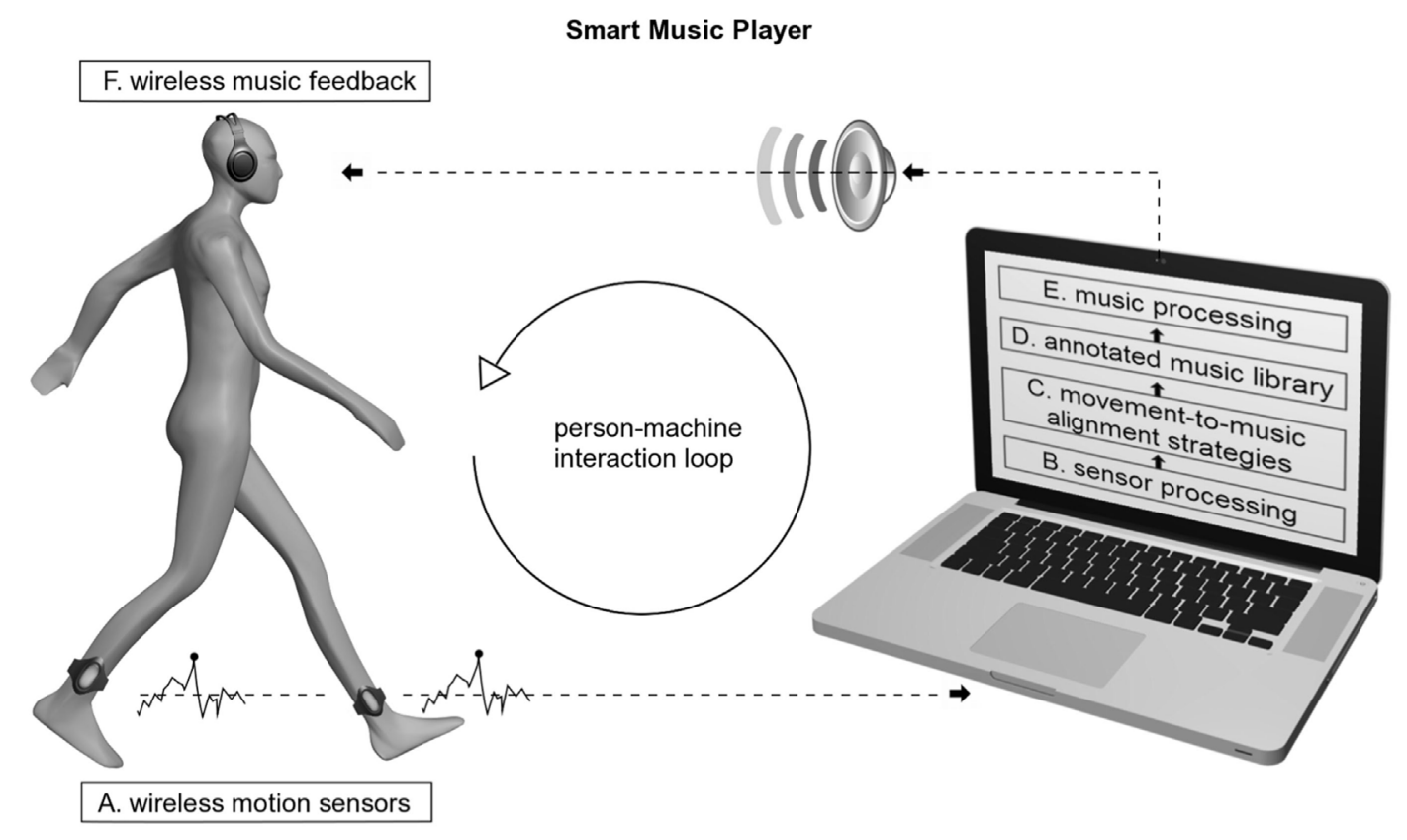

FIGURE 4 | Smart music player: person-machine interaction loop and the main components involved. Reproduced from Moens et al. (112).

be corrected using visual cues $(115,116)$. Multiple studies have shown that matching footsteps to visual cues such as equidistant horizontal lines along a walkway improves gait and reduces FOG in PD patients (117-119). Although visual cueing can be beneficial, replicating clinical scenarios would be unfeasible for patients who wish to train at home in a daily basis. Furthermore, as with auditory cueing, fixed walkway strips may be less effective than an interactive system that adjusts cueing based on the patient's movement and gait parameters. Instead, an ideal cueing system would involve adaptive feedback and include both visual 
and auditory stimuli. Immersive virtual reality (VR) technology could fill this gap by optimizing visually cued gait training. VR is an immersive and interactive computer-generated environment that simulates the real-world experience (120) and can be operated using a custom-made or commercially available headmounted display. The use of VR with visual cueing for clinical rehabilitation is still in its infancy, though multiple studies have found that in chronic stroke patients VR-based training improves cadence, step length, stride length, symmetry, and other gait parameters (120-123).

Recently, immersive VR was shown to be effective for gait rehabilitation in PD (124). The study uses a pair of VR glasses that projects a virtual checkered tile floor into the user's visual field. The user is instructed to walk along the floor, and the VR floor adapts to the user's body movements by simulating the visual effect of walking. Twenty PD patients with a mean age of 71.25 participated in the study. While wearing the device, the patients tried to match their steps with the adjacent tile to regulate their gait via the VR visual feedback. When cued by the VR display, the patients showed significant improvement in walking speed $(P=0.002)$ and stride length $(P=0.002)$ compared to baseline. Fifteen minutes post-training and without the device, the patients showed even greater improvements in walking speed $(P=0)$ and stride length $(P=0)$ compared to baseline (Figure 5).

Although these findings are promising, more well-controlled studies are needed to demonstrate the efficacy of VR-based therapies for PD. A potential expansion of VR gait training should involve adaptive, multisensory visual (e.g., virtual tiles or strips) and auditory (e.g., metronome and music) cues. Simultaneous multisensory cues could have a stronger combined effect than each cue alone. VR systems can be portable, enabling patients to train their gait in the comfort of their home. VR devices already have the computing capacity required for the integration of simultaneous adaptive cueing and can be internally processed or remotely processed in a smartphone connected to the VR

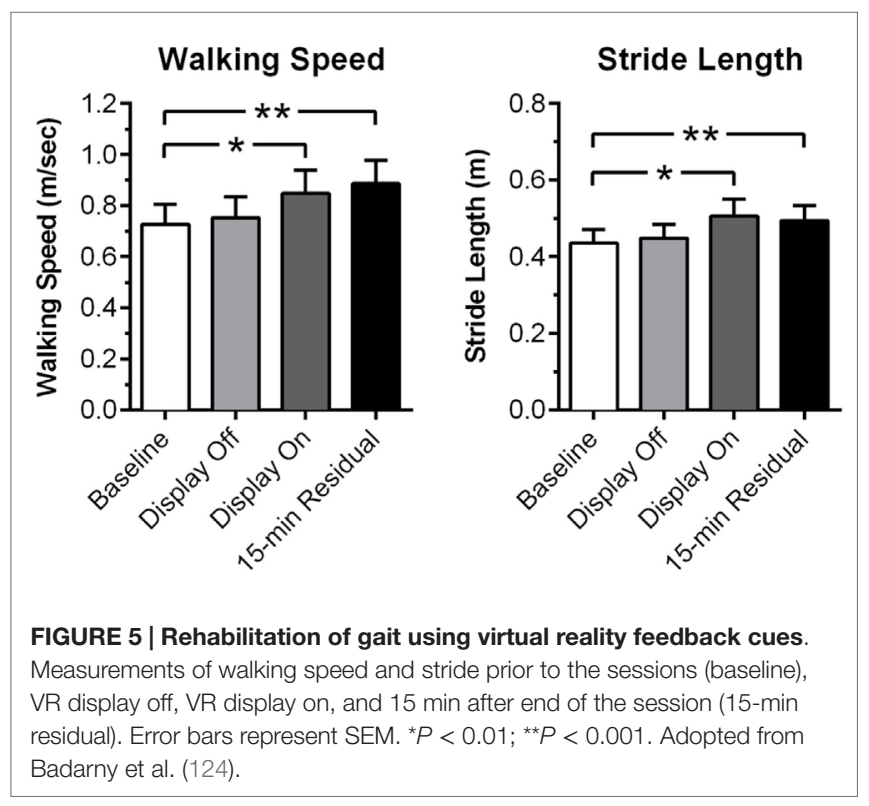

device via Bluetooth. Furthermore, VR devices are capable of measuring the users' performance via tracking technology (125), which would allow VR systems to provide feedback of the users' improved gait performance during and following training (126). Thus, a multisensory and adaptive VR device with performance tracking should be explored as a superior gait-training therapy.

\section{CONCLUSION}

Similar to how the metronome helps musicians maintain a steady tempo during a musical performance, RAS provides an effective approach for reducing gait impairments in PD patients. The efficacy of RAS reflects the overlapping neurological domains involved in gait and beat perception. Importantly, RAS is safe (127), inexpensive, non-invasive, and free of adverse health effects. One major limitation to most RAS methods is the fixedtempo design that requires increased cognitive demand and can negatively impact gait. However, an interactive cueing system that adapts to the patients' gait parameters may be able to resolve this limitation and maximize gait improvement from RAS. For RAS to be successful, the intervention should be initiated early in the progression of $\mathrm{PD}$ to maximize a participant's ability to adapt to the demands of the training before the development of cognitive impairment.

Further investigation of mechanisms of gait impairment in various parkinsonian disorders is needed. For example, an unresolved question is whether lower body parkinsonism, which is frequently associated with FOG, is a subtype of PD (37) or whether it represents a separate entity, such as vascular parkinsonism $(128,129)$, cortical Lewy Body disease (48), or atypical parkinsonism such as progressive supranuclear palsy or normal pressure hydrocephalus (1). The long-term impact of RAS on gait impairment and other motor and cognitive deficits should be objectively assessed by randomizing subjects to either participate in RAS by a trained therapist at least once a week for 6 months or participate in routine gait training. Novel methods and instruments, such as quantitative stepping-in-place with a concurrent mental task using a fourth generation iPod Touch sensor system (130), are needed to assess the effects of RAS on gait and mental function. The type of music and rhythm needed to optimize response to RAS should also be further evaluated. For example, in one study of healthy individuals' strikingly prominent (salient) commercially available music increased measures of cadence, velocity, and stride length, but simple music tempo did not (131). We suggest that different types of music, rather than the traditional rhythmic auditory cues, are carefully evaluated in patients with PD to determine which music most effectively improves PD-related gait disorders.

Another approach to gait rehabilitation is the use of VR for PD. While initial research to this immersive approach is promising, further studies are required and should integrate RAS. VR technology holds the potential to deliver more effective rhythmic cues by combining RAS and visual cueing, which we term rhythmic auditory and visual stimulation. With modern technology, VR-based rehabilitation could be made portable, and smartphones could be programed to process adaptive cue algorithms. Portability and 
ease of use could increase the frequency of gait-training sessions and improve compliance. Adaptive auditory and visual cueing could also be combined with tactile stimulation as a more salient gait therapy for PD patients. Concepts of tactile stimulation could be informed by recent innovations, such as the versatile extrasensory transducer (VEST), a non-invasive, low-cost vibratory VEST developed by Novich and Eagleman (132). Thus, RAS is a promising therapy for the gait impairments in $\mathrm{PD}$ and other movement disorders, and combining adaptive RAS with visual

\section{REFERENCES}

1. Jankovic J. Gait disorders. Neurol Clin (2015) 33(1):249-68. doi:10.1016/j. ncl.2014.09.007

2. de Dreu MJ, van der Wilk ASD, Poppe E, Kwakkel G, van Wegen EEH. Rehabilitation, exercise therapy and music in patients with Parkinson's disease: a meta-analysis of the effects of music-based movement therapy on walking ability, balance and quality of life. Parkinsonism Relat Disord (2012) 18:S114-9. doi:10.1016/S1353-8020(11)70036-0

3. Patel N, Jankovic J, Hallett M. Sensory aspects of movement disorders. Lancet Neurol (2014) 13(1):100-12. doi:10.1016/S1474-4422(13)70213-8

4. Paulson S, Bharucha J, Iyer V, Limb C, Tomaino C. Music and the mind: the magical power of sound. Ann N Y Acad Sci (2013) 1303:63-79. doi:10.1111/ nyas. 12183

5. Holmes D. Music therapy's breakthrough act. Lancet Neurol (2012) 11(6):486-7. doi:10.1016/S1474-4422(11)70294-0

6. Westfall SS. Gabby Giffords Says Music Therapy Has Helped Her Recover. People Magazine (2015).

7. Francois C, Grau-Sanchez J, Duarte E, Rodriguez-Fornells A. Musical training as an alternative and effective method for neuro-education and neuro-rehabilitation. Front Psychol (2015) 6:475. doi:10.3389/fpsyg.2015.00475

8. Sacks O. Musicophilia: Tales of Music and the Brain. New York, NY: Vintage Books (2008).

9. Jankovic J, Ashoori A. Movement disorders in musicians. Mov Disord (2008) 23(14):1957-65. doi:10.1002/mds.22255

10. Albanese A, Bhatia K, Bressman SB, Delong MR, Fahn S, Fung VS, et al. Phenomenology and classification of dystonia: a consensus update. Mov Disord (2013) 28(7):863-73. doi:10.1002/mds.25475

11. Kennedy M, Kennedy JB. The Concise Oxford Dictionary of Music. 5th ed. Oxford: Oxford University Press (2007).

12. Haueisen J, Knosche TR. Involuntary motor activity in pianists evoked by music perception. J Cogn Neurosci (2001) 13(6):786-92. doi:10.1162/08989290152541449

13. Bangert $M$, Altenmuller EO. Mapping perception to action in piano practice: a longitudinal DC-EEG study. BMC Neurosci (2003) 4:26. doi:10.1186/1471-2202-4-26

14. Baumann S, Koeneke S, Meyer M, Lutz K, Jancke L. A network for sensory-motor integration: what happens in the auditory cortex during piano playing without acoustic feedback? Ann N Y Acad Sci (2005) 1060:186-8. doi:10.1196/annals. 1360.038

15. Bangert M, Peschel T, Schlaug G, Rotte M, Drescher D, Hinrichs H, et al. Shared networks for auditory and motor processing in professional pianists: evidence from fMRI conjunction. Neuroimage (2006) 30(3):917-26. doi:10.1016/j.neuroimage.2005.10.044

16. Lahav A, Saltzman E, Schlaug G. Action representation of sound: audiomotor recognition network while listening to newly acquired actions. J Neurosci (2007) 27(2):308-14. doi:10.1523/JNEUROSCI.4822-06.2007

17. Zatorre RJ, Chen JL, Penhune VB. When the brain plays music: auditory-motor interactions in music perception and production. Nat Rev Neurosci (2007) 8(7):547-58. doi:10.1038/nrn2152

18. Chen JL, Penhune VB, Zatorre RJ. Listening to musical rhythms recruits motor regions of the brain. Cereb Cortex (2008) 18(12):2844-54. doi:10.1093/ cercor/bhn003

19. Thaut MH, McIntosh GC, Hoemberg V. Neurobiological foundations of neurologic music therapy: rhythmic entrainment and the motor system. Front Psychol (2014) 5:1185. doi:10.3389/fpsyg.2014.01185 and tactile cues in a VR device could further enhance the efficacy of this therapy.

\section{ACKNOWLEDGMENTS}

We would like to thank Amanda M. Buch, a research scientist at Columbia University Medical Center, for her editing and valuable comments. We also thank the National Parkinson Foundation for their support of the Baylor College of Medicine Center of Excellence.

20. Zivotofsky AZ, Hausdorff JM. The sensory feedback mechanisms enabling couples to walk synchronously: an initial investigation. J Neuroeng Rehabil (2007) 4:28. doi:10.1186/1743-0003-4-28

21. Nessler JA, De Leone CJ, Gilliland S. Nonlinear time series analysis of knee and ankle kinematics during side by side treadmill walking. Chaos (2009) 19(2):026104. doi:10.1063/1.3125762

22. Nessler JA, McMillan D, Schoulten M, Shallow T, Stewart B, De Leone C. Side by side treadmill walking with intentionally desynchronized gait. Ann Biomed Eng (2013) 41(8):1680-91. doi:10.1007/s10439-012-0657-6

23. Larsson M. Self-generated sounds of locomotion and ventilation and the evolution of human rhythmic abilities. Anim Cogn (2014) 17(1):1-14. doi:10.1007/s10071-013-0678-z

24. Fraisse P. Les Structures Rythmiques. Paris: Érasme (1956).

25. Fraisse P. Rhythm and tempo. In: Deutsch D, editor. The Psychology of Music. New York, NY: Academic Press (1982). p. 149-80.

26. Repp B. Subliminal temporal discrimination revealed in sensorimotor coordination. In: Desain P, Windsor L, editors. Rhythm Perception and Production. Lisse: Swets \& Zeitlinger Publishers (2000). p. 129-42.

27. van Noorden L, Moelants D. Resonance in the perception of musical pulse. J New Music Res (2010) 28(1):43-66. doi:10.1076/jnmr.28.1.43.3122

28. Moelants D. Preferred tempo reconsidered. In: Stevens C, Burnham D, McPherson G, Schubert E, Renwick J, editors. Proceedings of the 7th International Conference on Music Perception and Cognition. Adelaide: Causal Productions (2002). p. 580-3.

29. Whittle MW. Gait Analysis: An Introduction. Oxford: ButterworthHeinemann Ltd. (2007).

30. Van Dyck E, Moens B, Buhmann J, Demey M, Coorevits E, Dalla Bella S, et al. Spontaneous entrainment of running cadence to music tempo. Sports Med Open (2015) 1(1):15. doi:10.1186/s40798-015-0025-9

31. Wu LJ, Jankovic J. Runner's dystonia. J Neurol Sci (2006) 251(1-2):73-6. doi:10.1016/j.jns.2006.09.003

32. Hirtz D, Thurman DJ, Gwinn-Hardy K, Mohamed M, Chaudhuri AR, Zalutsky R. How common are the "common" neurologic disorders? Neurology (2007) 68(5):326-37. doi:10.1212/01.wnl.0000252807.38124.a3

33. Jankovic J. Parkinson's disease: clinical features and diagnosis. J Neurol Neurosurg Psychiatry (2008) 79(4):368-76. doi:10.1136/jnnp.2007.131045

34. Kalia LV, Lang AE. Parkinson's disease. Lancet (2015) 386(9996):896-912. doi:10.1016/S0140-6736(14)61393-3

35. Lees AJ. A modern perspective on the top 100 cited JNNP papers of all time the relevance of the Lewy body to the pathogenesis of idiopathic Parkinson's disease accuracy of clinical diagnosis of idiopathic Parkinson's disease. J Neurol Neurosurg Psychiatry (2012) 83(10):954-5. doi:10.1136/ jnnp-2012-302969

36. Factor SA, Higgins DS, Qian J. Primary progressive freezing gait: a syndrome with many causes. Neurology (2006) 66(3):411-4. doi:10.1212/01. wnl.0000196469.52995.ab

37. Thenganatt MA, Jankovic J. Parkinson disease subtypes. JAMA Neurol (2014) 71(4):499-504. doi:10.1001/jamaneurol.2013.6233

38. Arias P, Cudeiro J. Effects of rhythmic sensory stimulation (auditory, visual) on gait in Parkinson's disease patients. Exp Brain Res (2008) 186(4):589-601. doi:10.1007/s00221-007-1263-y

39. Bloem BR, Hausdorff JM, Visser JE, Giladi N. Falls and freezing of gait in Parkinson's disease: a review of two interconnected, episodic phenomena. Mov Disord (2004) 19(8):871-84. doi:10.1002/mds.20115

40. Grabli D, Karachi C, Welter ML, Lau B, Hirsch EC, Vidailhet M, et al. Normal and pathological gait: what we learn from Parkinson's disease. 
J Neurol Neurosurg Psychiatry (2012) 83(10):979-85. doi:10.1136/ jnnp-2012-302263

41. Blin O, Ferrandez AM, Serratrice G. Quantitative analysis of gait in Parkinson patients: increased variability of stride length. J Neurol Sci (1990) 98(1):91-7. doi:10.1016/0022-510X(90)90184-O

42. Morris ME, Iansek R, Matyas TA, Summers JJ. Ability to modulate walking cadence remains intact in Parkinson's disease. J Neurol Neurosurg Psychiatry (1994) 57(12):1532-4. doi:10.1136/jnnp.57.12.1532

43. Morris ME, Iansek R, Matyas TA, Summers JJ. Stride length regulation in Parkinson's disease. Normalization strategies and underlying mechanisms. Brain (1996) 119(Pt 2):551-68. doi:10.1093/brain/119.2.551

44. Thaut MH, McIntosh GC, Rice RR, Miller RA, Rathbun J, Brault JM. Rhythmic auditory stimulation in gait training for Parkinson's disease patients. Mov Disord (1996) 11(2):193-200. doi:10.1002/mds.870110213

45. Ebersbach G, Heijmenberg M, Kindermann L, Trottenberg T, Wissel J, Poewe W. Interference of rhythmic constraint on gait in healthy subjects and patients with early Parkinson's disease: evidence for impaired locomotor pattern generation in early Parkinson's disease. Mov Disord (1999) 14(4):619-25. doi:10.1002/1531-8257(199907)14:4<619::AID-MDS1011>3.0.CO;2-X

46. Hausdorff JM. Gait dynamics in Parkinson's disease: common and distinct behavior among stride length, gait variability, and fractal-like scaling. Chaos (2009) 19(2):026113. doi:10.1063/1.3147408

47. Tolleson CM, Dobolyi D, Roman OC, Kanoff K, Barton S, Wylie SA, et al. Dysrhythmia of timed movements in Parkinson's disease and freezing of gait. Brain Res (2015). doi:10.1016/j.brainres.2015.07.041

48. Virmani T, Moskowitz CB, Vonsattel JP, Fahn S. Clinicopathological characteristics of freezing of gait in autopsy-confirmed Parkinson's disease. Mov Disord (2015). doi:10.1002/mds.26346

49. Perez-Lloret S, Negre-Pages L, Damier P, Delval A, Derkinderen P, Destee A, et al. Prevalence, determinants, and effect on quality of life of freezing of gait in Parkinson disease. JAMA Neurol (2014) 71(7):884-90. doi:10.1001/ jamaneurol.2014.753

50. Shine JM, Matar E, Ward PB, Frank MJ, Moustafa AA, Pearson M, et al. Freezing of gait in Parkinson's disease is associated with functional decoupling between the cognitive control network and the basal ganglia. Brain (2013) 136(Pt 12):3671-81. doi:10.1093/brain/awt272

51. Szewczyk-Krolikowski K, Menke RA, Rolinski M, Duff E, Salimi-Khorshidi G, Filippini N, et al. Functional connectivity in the basal ganglia network differentiates PD patients from controls. Neurology (2014) 83(3):208-14. doi:10.1212/WNL.0000000000000592

52. Bohnen NI, Frey KA, Studenski S, Kotagal V, Koeppe RA, Constantine GM, et al. Extra-nigral pathological conditions are common in Parkinson's disease with freezing of gait: an in vivo positron emission tomography study. Mov Disord (2014) 29(9):1118-24. doi:10.1002/mds.25929

53. Bella SD, Benoit CE, Farrugia N, Schwartze M, Kotz SA. Effects of musically cued gait training in Parkinson's disease: beyond a motor benefit. Ann N Y Acad Sci (2015) 1337:77-85. doi:10.1111/nyas.12651

54. Fasano A, Daniele A, Albanese A. Treatment of motor and non-motor features of Parkinson's disease with deep brain stimulation. Lancet Neurol (2012) 11(5):429-42. doi:10.1016/S1474-4422(12)70049-2

55. Lozano AM, Snyder BJ. Deep brain stimulation for parkinsonian gait disorders. J Neurol (2008) 255(Suppl 4):30-1. doi:10.1007/ s00415-008-4005-6

56. Ferraye MU, Debu B, Fraix V, Goetz L, Ardouin C, Yelnik J, et al. Effects of pedunculopontine nucleus area stimulation on gait disorders in Parkinson's disease. Brain (2010) 133(Pt 1):205-14. doi:10.1093/brain/awp229

57. Jones CR, Jahanshahi M. Motor and perceptual timing in Parkinson's disease. Adv Exp Med Biol (2014) 829:265-90. doi:10.1007/978-1-4939-1782-2_14

58. Gomez J, Jesus Marin-Mendez J, Molero P, Atakan Z, Ortuno F. Time perception networks and cognition in schizophrenia: a review and a proposal. Psychiatry Res (2014) 220(3):737-44. doi:10.1016/j.psychres.2014.07.048

59. Parker KL, Lamichhane D, Caetano MS, Narayanan NS. Executive dysfunction in Parkinson's disease and timing deficits. Front Integr Neurosci (2013) 7(75):75. doi:10.3389/fnint.2013.00075

60. Malapani C, Rakitin B, Levy R, Meck WH, Deweer B, Dubois B, et al. Coupled temporal memories in Parkinson's disease: a dopamine-related dysfunction. $J$ Cogn Neurosci (1998) 10(3):316-31. doi:10.1162/089892998562762

61. Drew MR, Simpson EH, Kellendonk C, Herzberg WG, Lipatova O, Fairhurst S, et al. Transient overexpression of striatal D2 receptors impairs operant motivation and interval timing. JNeurosci (2007) 27(29):7731-9. doi:10.1523/ JNEUROSCI.1736-07.2007

62. Eagleman DM, Tse PU, Buonomano D, Janssen P, Nobre AC, Holcombe AO. Time and the brain: how subjective time relates to neural time. J Neurosci (2005) 25(45):10369-71. doi:10.1523/JNEUROSCI.3487-05.2005

63. Parsons BD, Gandhi S, Aurbach EL, Williams N, Williams M, Wassef A, et al. Lengthened temporal integration in schizophrenia. Neuropsychologia (2013) 51(2):372-6. doi:10.1016/j.neuropsychologia.2012.11.008

64. Coull J, Nobre A. Dissociating explicit timing from temporal expectation with fMRI. Curr Opin Neurobiol (2008) 18(2):137-44. doi:10.1016/j. conb.2008.07.011

65. Piras F, Coull JT. Implicit, predictive timing draws upon the same scalar representation of time as explicit timing. PLoS One (2011) 6(3):e18203. doi:10.1371/journal.pone.0022514

66. Artieda J, Pastor MA, Lacruz F, Obeso JA. Temporal discrimination is abnormal in Parkinson's disease. Brain (1992) 115(1):199-210. doi:10.1093/ brain/115.1.199

67. Harrington DL, Haaland KY,HermanowitzN. Temporal processing in thebasal ganglia. Neuropsychology (1998) 12(1):3-12. doi:10.1037/0894-4105.12.1.3

68. Smith JG, Harper DN, Gittings D, Abernethy D. The effect of Parkinson's disease on time estimation as a function of stimulus duration range and modality. Brain Cogn (2007) 64(2):130-43. doi:10.1016/j.bandc.2007.02.001

69. Grahn JA, Brett M. Impairment of beat-based rhythm discrimination in Parkinson's disease. Cortex (2009) 45(1):54-61. doi:10.1016/j. cortex.2008.01.005

70. Bares M, Lungu O, Liu T, Waechter T, Gomez CM, Ashe J. Impaired predictive motor timing in patients with cerebellar disorders. Exp Brain Res (2007) 180(2):355-65. doi:10.1007/s00221-007-0857-8

71. Beudel M, Galama S, Leenders KL, de Jong BM. Time estimation in Parkinson's disease and degenerative cerebellar disease. Neuroreport (2008) 19(10):1055-8. doi:10.1097/WNR.0b013e328303b7b9

72. Merchant H, Grahn J, Trainor L, Rohrmeier M, Fitch WT. Finding the beat: a neural perspective across humans and non-human primates. Philos Trans $R$ Soc Lond B Biol Sci (2015) 370(1664):20140093. doi:10.1098/rstb.2014.0093

73. Coull JT, Cheng RK, Meck WH. Neuroanatomical and neurochemical substrates of timing. Neuropsychopharmacology (2011) 36(1):3-25. doi:10.1038/ npp. 2010.113

74. Bengtsson SL, Ullen F, Ehrsson HH, Hashimoto T, Kito T, Naito E, et al. Listening to rhythms activates motor and premotor cortices. Cortex (2009) 45(1):62-71. doi:10.1016/j.cortex.2008.07.002

75. Schaefer RS, Overy K. Motor responses to a steady beat. Ann N Y Acad Sci (2015) 1337:40-4. doi:10.1111/nyas.12717

76. Pastor MA, Artieda J, Jahanshahi M, Obeso JA. Time estimation and reproduction is abnormal in Parkinson's disease. Brain (1992) 115(1):211-25. doi:10.1093/brain/115.1.211

77. Ivry RB. The representation of temporal information in perception and motor control. Curr Opin Neurobiol (1996) 6(6):851-7. doi:10.1016/ S0959-4388(96)80037-7

78. Ivry RB, Spencer RM. The neural representation of time. Curr Opin Neurobiol (2004) 14(2):225-32. doi:10.1016/j.conb.2004.03.013

79. Grahn JA, Rowe JB. Finding and feeling the musical beat: striatal dissociations between detection and prediction of regularity. Cereb Cortex (2013) 23(4):913-21. doi:10.1093/cercor/bhs083

80. Meck WH. Affinity for the dopamine D2 receptor predicts neuroleptic potency in decreasing the speed of an internal clock. Pharmacol Biochem Behav (1986) 25(6):1185-9. doi:10.1016/0091-3057(86)90109-7

81. Drew MR, Fairhurst S, Malapani C, Horvitz JC, Balsam PD. Effects of dopamine antagonists on the timing of two intervals. Pharmacol Biochem Behav (2003) 75(1):9-15. doi:10.1016/S0091-3057(03)00036-4

82. Buhusi CV, Meck WH. What makes us tick? Functional and neural mechanisms of interval timing. Nat Rev Neurosci (2005) 6(10):755-65. doi:10.1038/ nrn1764

83. Meck WH. Neuroanatomical localization of an internal clock: a functional link between mesolimbic, nigrostriatal, and mesocortical dopaminergic systems. Brain Res (2006) 1109(1):93-107. doi:10.1016/j.brainres.2006.06.031

84. Buhusi CV, Meck WH. Relativity theory and time perception: single or multiple clocks? PLoS One (2009) 4(7):e6268. doi:10.1371/journal.pone.0006268

85. Lake JI, Meck WH. Differential effects of amphetamine and haloperidol on temporal reproduction: dopaminergic regulation of attention and 
clock speed. Neuropsychologia (2013) 51(2):284-92. doi:10.1016/j. neuropsychologia.2012.09.014

86. O'Boyle DJ, Freeman JS, Cody FW. The accuracy and precision of timing of self-paced, repetitive movements in subjects with Parkinson's disease. Brain (1996) 119(Pt 1):51-70. doi:10.1093/brain/119.1.51

87. Harrington DL, Haaland KY. Neural underpinnings of temporal processing: a review of focal lesion, pharmacological, and functional imaging research. Rev Neurosci (1999) 10(2):91-116. doi:10.1515/REVNEURO.1999.10.2.91

88. Schwartze M, Keller PE, Patel AD, Kotz SA. The impact of basal ganglia lesions on sensorimotor synchronization, spontaneous motor tempo, and the detection of tempo changes. Behav Brain Res (2011) 216(2):685-91. doi:10.1016/j.bbr.2010.09.015

89. Sen S, Kawaguchi A, Truong Y, Lewis MM, Huang X. Dynamic changes in cerebello-thalamo-cortical motor circuitry during progression of Parkinson's disease. Neuroscience (2010) 166(2):712-9. doi:10.1016/j. neuroscience.2009.12.036

90. Parsons BD, Novich SD, Eagleman DM. Motor-sensory recalibration modulates perceived simultaneity of cross-modal events at different distances. Front Psychol (2013) 4:46. doi:10.3389/fpsyg.2013.00046

91. Leuthold H, Jentzsch I. Neural correlates of advance movement preparation: a dipole source analysis approach. Brain Res Cogn Brain Res (2001) 12(2):207-24. doi:10.1016/S0926-6410(01)00052-0

92. Cajal R, Swanson N, Swanson L. Histology of the Nervous System of Man and Vertebrates. New York, NY: Oxford University Press (1953).

93. Nieuwboer A, Rochester L, Müncks L, Swinnen SP. Motor learning in Parkinson's disease: limitations and potential for rehabilitation. Parkinsonism Relat Disord (2009) 15:S53-8. doi:10.1016/j.parkreldis.2008.03.003

94. Nombela C, Hughes LE, Owen AM, Grahn JA. Into the groove: can rhythm influence Parkinson's disease? Neurosci Biobehav Rev (2013) 37(10 Pt 2):2564-70. doi:10.1016/j.neubiorev.2013.08.003

95. McIntosh GC, Brown SH, Rice RR, Thaut MH. Rhythmic auditory-motor facilitation of gait patterns in patients with Parkinson's disease. J Neurol Neurosurg Psychiatry (1997) 62(1):22-6. doi:10.1136/jnnp.62.1.22

96. Styns F, van Noorden L, Moelants D, Leman M. Walking on music. Hum Mov Sci (2007) 26(5):769-85. doi:10.1016/j.humov.2007.07.007

97. Wittwer JE, Webster KE, Hill K. Music and metronome cues produce different effects on gait spatiotemporal measures but not gait variability in healthy older adults. Gait Posture (2013) 37(2):219-22. doi:10.1016/j. gaitpost.2012.07.006

98. Thaut $\mathrm{MH}$, Miltner R, Lange HW, Hurt CP, Hoemberg V. Velocity modulation and rhythmic synchronization of gait in Huntington's disease. Mov Disord (1999) 14(5):808-19. doi:10.1002/1531-8257(199909)14:5<808::AID-MDS1014>3.0.CO;2-J

99. Leow LA, Parrott T, Grahn JA. Individual differences in beat perception affect gait responses to low- and high-groove music. Front Hum Neurosci (2014) 8:811. doi:10.3389/fnhum.2014.00811

100. Leow LA, Rinchon C, Grahn J. Familiarity with music increases walking speed in rhythmic auditory cuing. Ann N Y Acad Sci (2015) 1337:53-61. doi:10.1111/nyas. 12658

101. Lopez WO, Higuera CA, Fonoff ET, Souza Cde O, Albicker U, Martinez JA. Listenmee and Listenmee smartphone application: synchronizing walking to rhythmic auditory cues to improve gait in Parkinson's disease. Hum Mov Sci (2014) 37:147-56. doi:10.1016/j.humov.2014.08.001

102. Benoit CE, Dalla Bella S, Farrugia N, Obrig H, Mainka S, Kotz SA. Musically cued gait-training improves both perceptual and motor timing in Parkinson's disease. Front Hum Neurosci (2014) 8:494. doi:10.3389/ fnhum.2014.00494

103. Pecenka N, Engel A, Keller PE. Neural correlates of auditory temporal predictions during sensorimotor synchronization. Front Hum Neurosci (2013) 7:380. doi:10.3389/fnhum.2013.00380

104. Rochester L, Hetherington V, Jones D, Nieuwboer A, Willems AM, Kwakkel G, et al. Attending to the task: interference effects of functional tasks on walking in Parkinson's disease and the roles of cognition, depression, fatigue, and balance. Arch Phys Med Rehabil (2004) 85(10):1578-85. doi:10.1016/j. apmr.2004.01.025

105. Yogev G, Giladi N, Peretz C, Springer S, Simon ES, Hausdorff JM. Dual tasking, gait rhythmicity, and Parkinson's disease: which aspects of gait are attention demanding? Eur J Neurosci (2005) 22(5):1248-56. doi:10.1111/j.1460-9568.2005.04298.x
106. Rochester L, Nieuwboer A, Baker K, Hetherington V, Willems AM, Chavret F, et al. The attentional cost of external rhythmical cues and their impact on gait in Parkinson's disease: effect of cue modality and task complexity. $J$ Neural Transm (2007) 114(10):1243-8. doi:10.1007/s00702-007-0756-y

107. Hausdorff JM, Purdon PL, Peng CK, Ladin Z, Wei JY, Goldberger AL. Fractal dynamics of human gait: stability of long-range correlations in stride interval fluctuations. J Appl Physiol (1985) (1996) 80(5):1448-57.

108. Hove MJ, Keller PE. Impaired movement timing in neurological disorders: rehabilitation and treatment strategies. Ann N Y Acad Sci (2015) 1337:111-7. doi:10.1111/nyas. 12615

109. Hove MJ, Suzuki K, Uchitomi H, Orimo S, Miyake Y. Interactive rhythmic auditory stimulation reinstates natural $1 / \mathrm{f}$ timing in gait of Parkinson's patients. PLoS One (2012) 7(3):e32600. doi:10.1371/journal.pone.0032600

110. Herman T, Giladi N, Gurevich T, Hausdorff JM. Gait instability and fractal dynamics of older adults with a "cautious" gait: why do certain older adults walk fearfully? Gait Posture (2005) 21(2):178-85. doi:10.1016/ S0966-6362(05)80311-X

111. Moens B, Leman M. Alignment strategies for the entrainment of music and movement rhythms. Ann N Y Acad Sci (2015) 1337:86-93. doi:10.1111/ nyas. 12647

112. Moens B, Muller C, van Noorden L, Franek M, Celie B, Boone J, et al. Encouraging spontaneous synchronisation with D-Jogger, an adaptive music player that aligns movement and music. PLoS One (2014) 9(12):e114234. doi:10.1371/journal.pone.0114901

113. Azulay JP, Mesure S, Amblard B, Pouget J. Increased visual dependence in Parkinson's disease. Percept Mot Skills (2002) 95(3 Pt 2):1106-14. doi:10.2466/ PMS.95.7.1106-1114

114. Caudron S, Guerraz M, Eusebio A, Gros JP, Azulay JP, Vaugoyeau M. Evaluation of a visual biofeedback on the postural control in Parkinson's disease. Neurophysiol Clin (2014) 44(1):77-86. doi:10.1016/j.neucli.2013.10.134

115. Adamovich SV, Berkinblit MB, Hening W, Sage J, Poizner H. The interaction of visual and proprioceptive inputs in pointing to actual and remembered targets in Parkinson's disease. Neuroscience (2001) 104(4):1027-41. doi:10.1016/ S0306-4522(01)00099-9

116. Keijsers NL, Admiraal MA, Cools AR, Bloem BR, Gielen CC. Differential progression of proprioceptive and visual information processing deficits in Parkinson's disease. Eur J Neurosci (2005) 21(1):239-48. doi:10.1111/j.1460-9568.2004.03840.x

117. Jiang Y, Norman KE. Effects of visual and auditory cues on gait initiation in people with Parkinson's disease. Clin Rehabil (2006) 20(1):36-45. doi:10.11 91/0269215506cr925oa

118. Lee SJ, Yoo JY, Ryu JS, Park HK, Chung SJ. The effects of visual and auditory cues on freezing of gait in patients with Parkinson disease. Am J Phys Med Rehabil (2012) 91(1):2-11. doi:10.1097/PHM.0b013e3182745a04

119. Luessi F, Mueller LK, Breimhorst M, Vogt T. Influence of visual cues on gait in Parkinson's disease during treadmill walking at multiple velocities. J Neurol Sci (2012) 314(1-2):78-82. doi:10.1016/j.jns.2011.10.027

120. Darekar A, McFadyen BJ, Lamontagne A, Fung J. Efficacy of virtual reality-based intervention on balance and mobility disorders post-stroke: a scoping review. J Neuroeng Rehabil (2015) 12(1):46. doi:10.1186/s12984-015-0035-3

121. Kim JH, Jang SH, Kim CS, Jung JH, You JH. Use of virtual reality to enhance balance and ambulation in chronic stroke: a double-blind, randomized controlled study. Am J Phys Med Rehabil (2009) 88(9):693-701. doi:10.1097/ PHM.0b013e3181b811e3

122. Lewek MD, Feasel J, Wentz E, Brooks FP Jr, Whitton MC. Use of visual and proprioceptive feedback to improve gait speed and spatiotemporal symmetry following chronic stroke: a case series. Phys Ther (2012) 92(5):748-56. doi:10.2522/ptj.20110206

123. Cho KH, Lee WH. Virtual walking training program using a real-world video recording for patients with chronic stroke: a pilot study. Am J Phys Med Rehabil (2013) 92(5):371-80; quiz 380-372, 458. doi:10.1097/ PHM.0b013e31828cd5d3

124. Badarny S, Aharon-Peretz J, Susel Z, Habib G, Baram Y. Virtual reality feedback cues for improvement of gait in patients with Parkinson's disease. Tremor Other Hyperkinet Mov (N Y) (2014) 4:225. doi:10.7916/ D8V69GM4

125. Rizzo AA, Bowerly T, Buckwalter JG, Klimchuk D, Mitura R, Parsons TD. A virtual reality scenario for all seasons: the virtual classroom. CNS Spectr (2006) 11(1):35-44. doi:10.1017/S1092852900024196 
126. Lange B, Koenig S, Chang CY, McConnell E, Suma E, Bolas M, et al. Designing informed game-based rehabilitation tasks leveraging advances in virtual reality. Disabil Rehabil (2012) 34(22):1863-70. doi:10.3109/0963828 8.2012.670029

127. de Bruin N, Doan JB, Turnbull G, Suchowersky O, Bonfield S, Hu B, et al. Walking with music is a safe and viable tool for gait training in Parkinson's disease: the effect of a 13-week feasibility study on single and dual task walking. Parkinsons Dis (2010) 2010:483530. doi:10.4061/2010/483530

128. FitzGerald PM, Jankovic J. Lower body parkinsonism: evidence for vascular etiology. Mov Disord. (1989) 4(3):249-60.

129. Korczyn AD. Vascular parkinsonism - characteristics, pathogenesis and treatment. Nat Rev Neurol. (2015) 11(6):319-26.

130. Chomiak T, Pereira FV, Meyer N, de Bruin N, Derwent L, Luan K, et al. A new quantitative method for evaluating freezing of gait and dual-attention task deficits in Parkinson's disease. J Neural Transm. (2015) 122(11):1523-31.
131. de Bruin N, Kempster C, Doucette A, Doan JB, Hu B, Brown LA. The effects of music salience on the gait performance of young adults. J Music Ther (2015).

132. Novich SD, Eagleman DM. Using space and time to encode vibrotactile information: toward an estimate of the skin's achievable throughput. Exp Brain Res (2015) 233(10):2777-88. doi:10.1007/s00221-015-4346-1

Conflict of Interest Statement: The authors declare that the research was conducted in the absence of any commercial or financial relationships that could be construed as a potential conflict of interest.

Copyright (C) 2015 Ashoori, Eagleman and Jankovic. This is an open-access article distributed under the terms of the Creative Commons Attribution License (CC BY). The use, distribution or reproduction in other forums is permitted, provided the original author(s) or licensor are credited and that the original publication in this journal is cited, in accordance with accepted academic practice. No use, distribution or reproduction is permitted which does not comply with these terms. 